\title{
ON RELATIVE GROTHENDIECK RINGS
}

\author{
BY ANDREAS DRESS
}

\author{
Communicated by Hyman Bass, April 21, 1969
}

Let $K$ be a field of characteristic $p(\neq 0$, to exclude trivial cases) and let $G$ be a finite goup. A $K G$-module $M$ is a finite dimensional $K$-vector space, on which $G$ acts $K$-linearly from the left.

The Green ring $a(G)$ of $G$ (w.r.t. $K$ ) is the free abelian group, spanned by the isomorphism classes of indecomposable $K G$-modules, with the multiplication induced from the tensor product $\oplus_{K}$ of $K G$ modules (see [4]).

If $U \leqq G$ one has a restriction map $a(G) \rightarrow a(U)$, induced from restricting the action of $G$ on a $K G$-module $M$ to $U$, thus getting a $K U$ module $\left.M\right|_{v}$. Let $\mathfrak{u}$ be a family of subgroups of $G$. An exact sequence

$$
E: 0 \rightarrow M^{\prime} \rightarrow M \rightarrow M^{\prime \prime} \rightarrow 0
$$

is said to be $\mathfrak{u}$-split, if

$$
\left.E\right|_{U}:\left.\left.\left.0 \rightarrow M^{\prime}\right|_{U} \rightarrow M\right|_{U} \rightarrow M^{\prime \prime}\right|_{U} \rightarrow 0
$$

is a split exact sequence of $K U$-modules for any $U \in \mathfrak{U}$.

For any $\mathfrak{u}$-split exact sequence $E$ of $K G$-modules define $\chi_{E}=M$ $-M^{\prime}-M^{\prime \prime}$ to be its Euler characteristic in $a(G)$. Write $i(G, \mathfrak{u})$ for the linear span of the elements $\chi_{E} \in a(G)$, where $E$ runs through all $\mathfrak{u}$-split exact sequences of $K G$-modules. $i(G, \mathfrak{u})$ is an ideal in $a(G)$ and $a(G, \mathfrak{u})=a(G) / i(G, \mathfrak{u})$ the Grothendieck ring of $G$ relative to $\mathfrak{U}$ (see [1], [6]).

Lemma 1. Let $\mathfrak{U}_{1}, \mathfrak{U}_{2}$ be two families of subgroups of $G$. Then the multiplication map $a(G) \times a(G) \rightarrow a(G)$ sends $i\left(G, \mathfrak{u}_{1}\right) \times i\left(G, \mathfrak{U}_{2}\right)$ into $i\left(G, \mathfrak{U}_{1} \cup \mathfrak{u}_{2}\right)$.

Proof. If $E_{i}: 0 \rightarrow M_{i}^{\prime} \rightarrow M_{i} \rightarrow M_{i}^{\prime \prime} \rightarrow 0$ is exact and $\mathfrak{U}_{i}$-split, then the tensor product of these two complexes $E_{1}, E_{2}$ is exact and $\mathfrak{U}_{1} \cup \mathfrak{u}_{2^{-}}$ split, therefore $\chi_{E_{i} \otimes E_{2}}=\chi_{B_{i}} \cdot \chi_{E_{2}} \in i\left(G, \mathfrak{U}_{1} \cup \mathfrak{u}_{2}\right)$.

An $K G$-module $M$ is $\mathfrak{U}$-projective, if $M$ is a direct summand in $\oplus_{v \in \mathfrak{u}}\left(\left.M\right|_{U}\right)^{U \rightarrow G}$ (see [3]), where for a $K U$-module $N$ we write $N^{U \rightarrow a}$ for the induced $K G$-module $K G \otimes_{K U} N$.

Write $k(G, \mathfrak{u})$ for the linear span of the $\mathfrak{U}$-projective modules in $a(G)$. The canonical epimorphism $a(G) \rightarrow a(G, \mathfrak{u})$ induces a map $\kappa: k(G, \mathfrak{u}) \rightarrow a(G, \mathfrak{u})$, which has also been called the Cartan map (see $[1],[6],[7])$. 
Theorem 1. The Cartan map $\kappa: k(G, \mathfrak{u}) \rightarrow a(G, \mathfrak{u})$ is monic and its cokernel is a p-power-torsion-module.

Corollary 1. All torsion in $a(G, \mathfrak{u}$ ) (if there is any) is p-powertorsion.

Proof. If $\operatorname{Tor}(a(G, \mathfrak{u}))=T$ is the torsion submodule of $a(G, \mathfrak{u})$, then $T \cap \kappa(k(G, \mathfrak{U}))=0$ because $\kappa$ is injective and $k(G, \mathfrak{U}) \subseteq a(G)$ torsion free. Thus $T$ maps injectively into cokernel $\kappa$. Now define $\mathscr{Z}=\left\{V \leqq G \mid V_{p} \unlhd V, V / V_{p}\right.$ cyclic and $V_{p} \subseteq U$ for some $\left.U \in \mathfrak{U}\right\}, V_{p}$ the $p$-Sylow subgroup of $V$. For $V \in \mathbb{Z} \mathfrak{U}$ the restriction map $a(G) \rightarrow a(V)$ factors over $a(G, \mathfrak{u})$.

THEOREM 2. The kernel of the product of the restriction maps:

is exactly $\operatorname{Tor}(a(G, \mathfrak{u}))$.

$$
a(G, \mathfrak{u}) \rightarrow \prod_{V \in \mathfrak{g} \mathfrak{u}} a(V)
$$

COROLLARY 2. If $G$ is a p-group, then the product of the restriction maps $a(G, \mathfrak{u}) \rightarrow \sum_{U \in \mathfrak{u}} a(U)$ is exactly $\operatorname{Tor}(a(G, \mathfrak{u}))$.

These results generalize some part of the more complete results, obtained in a more special case, in [8, Theorems $4,5,6]$.

CoRollary 3. (a) If $M$ is $\mathfrak{U}$-projective, then there exists a number $n$ and $K V$-modules $M_{1}(V), M_{2}(V)(V \in \mathbb{Z})$ with

$$
\begin{aligned}
& M \oplus M \oplus \cdots \oplus M \oplus \oplus M_{1}(V)^{V \rightarrow G} \cong \oplus M_{2}(V)^{V \rightarrow G} . \\
& \text { (n-times) } \quad v \in \mathfrak{3 u} \quad v \in \mathfrak{Z} \mathfrak{u}
\end{aligned}
$$

(b) If $M_{1}, M_{2}$ are two $\mathfrak{U}$-projective $K G$-modules, then $M_{1} \cong M_{2}$ if and only if $\left.\left.M_{1}\right|_{v} \cong M_{2}\right|_{v}$ for all $V \in \Re \mathfrak{B}$.

REMARK 1. One can also prove Corollary 3 more directly with an induction argument, using Brauer's theory of modular characters $(\mathfrak{U}=\{E\})$ for the start of the induction and Green's Transfer theorem (see [5]) for the induction step.

REMARK 2. Corollary 3, (b) implies easily Theorem 3.8 in [7].

REMARK 3. If $V \in \mathfrak{M}$, one can find two $\mathfrak{U}$-projective $K G$-modules $M_{1}, M_{2}$ with $\left.\left.M_{1}\right|_{H} \cong M_{2}\right|_{H}$ for any $H \leqq G$, which does not contain any conjugate of $V$, but $\left.M_{1}\right|_{V} \neq\left. M_{2}\right|_{v}$ and a fortiori $M_{1} \neq M_{2}$. Therefore-up to conjugation-the maximal elements in $3 \mathfrak{U}$ is the smallest (i.e. best) possible family of subgroups in $G$, for which Corollary 3 and thus also Theorem 2 -can hold.

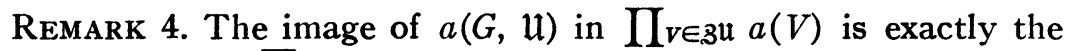
image of $a(G)$ in $\sum_{V \in \mathfrak{B l}} a(V)$ and this is to some extent described 
in [2]. It seems that in this rather general situation not much more can be done. For more precise information in some special cases see $[7],[8]$.

Theorems 1 and 2 are easy consequences of the following

TheOREM 3. With $\mathfrak{S} \mathfrak{U}=\left\{V \leqq G \mid V_{p} \unlhd G, V / V_{p}\right.$ hyperelementary, $V_{p} \subseteq U$ for some $\left.U \in \mathfrak{U}\right\}$ there exist a p-power $p^{n}$ and integral numbers $n_{V}(V \in \mathfrak{S} \mathfrak{U})$ with $p^{n} \cdot 1=\sum_{V \in \mathfrak{s u}} n_{V} \cdot K[G / V]$ in $a(G, \mathfrak{u})$ with $K[G / V]$ the $K G$-module spanned by the cosets $G / V$.

Using Corollary 3 for $\mathfrak{U}=\{G\}$ Theorem 3 reduces itself easily to the case $G_{p} \unlhd G, G_{p}$ elementary abelian and $\mathfrak{U}=\mathfrak{M}\left(G_{p}\right)$, the family of maximal subgroups in $G_{p}$. In this special case one can explictly construct the numbers $p^{n}, n_{V}(V \in \mathfrak{S} \mathfrak{U})$, using constructions based on Lemma 1.

Theorem 3 implies also a statement similar to Corollary 3 : For any $\mathfrak{U}$-projective $K G$-module $M$ there exists a $p$-power $p^{n}$ and $K V$-modules $M_{1}(V), M_{2}(V)(V \in \mathfrak{S} \mathfrak{U})$ with

$$
\begin{aligned}
& M \oplus \cdots \oplus M \oplus \underset{V \in \mathfrak{W u}}{\oplus} M_{1}(V)^{V \rightarrow G} \cong \underset{V \in \mathfrak{W} \mathfrak{u}}{\bigoplus} M_{2}(V)^{V \rightarrow G} . \\
& \quad\left(p^{n} \text { times }\right)
\end{aligned}
$$

One may also assume either $M_{1}(V)=0$ or $M_{2}(V)=0$ for any $V \in \mathfrak{S} \mathfrak{U}$ (the same remark holds for Corollary 3 ).

REMARK 5. If $R$ is any commutative ring, one may as well form the relative Grothendieck ring $a(G, \mathfrak{u} ; R)$, spanned by the isomorphism classes of finitely generated $R G$-modules, modulo the span of the Euler characteristics of $\mathfrak{U}$-split exact sequences of $R G$-modules. One has also the notion of $\mathfrak{U}$-projective $R G$-modules [3] and a Cartan map $\kappa: k(G, \mathfrak{u} ; R) \rightarrow a(G, \mathfrak{u} ; R)$. One can still prove that the kernel of $\kappa$ is a torsion submodule of $k(G, \mathfrak{u} ; R)$, probably it is even injective, but the cokernel is no longer a torsion module. (Counterexample: $G$ cyclic of order $p, \mathfrak{U}=\{E\}, R=\hat{\boldsymbol{Z}}_{p}$.) But the following form of Theorem 2 may still be true:

Conjecture. With $\mathfrak{C}_{R} \mathfrak{U}=\{V \leq G \mid \exists N \unlhd V, V / N$ cyclic, $N \subseteq U$ for some $U \in \mathfrak{U}$ and $N$ a $p$-group for some $p$ with $p R \neq R$, i.e. $p$ not a unit in $R$ \} the map

$$
a(G, \mathfrak{u} ; R) \rightarrow \prod_{V \in \mathbb{E}_{\mathbb{R} \mathfrak{u}}} a(V, V \cap \mathfrak{u} ; R)
$$

has torsion kernel with

$$
V \cap \mathfrak{u}=\left\{V \cap g U g^{-1} \mid g \in G, U \in \mathfrak{u}\right\} .
$$


For $\mathfrak{U}=\{E\}$ this is a Corollary to the results of Swan [9], for $\mathfrak{U}=\{G\}$ this is the principal result in [2].

REMARK 6 . There is another perhaps interesting application of the construction, used in the proof of Lemma 1: Let $R$ be a commutative noetherian ring, whose maximal ideal spectrum is of dimension $\leqq n$. Let $G$ be a finite group and consider the Grothendieck ring $a(G ; R)$ $=a(G,\{G\} ; R)$ in the above notation. For any maximal ideal $\mathfrak{m}$ one has a homomorphism $a(G ; R) \rightarrow a\left(G, R_{\mathfrak{m}}\right)$ with kernel say $i(G ; R, \mathfrak{m})$. Then $\bigcap_{\mathfrak{m}} i(G ; R, \mathfrak{m})=i(G, R)$ (where $\mathfrak{m}$ runs through all maximal ideals in $R$ ) is nilpotent of order $n+1$, i.e. $i(G, R)^{n+1}=0$.

Especially if $R$ is a Dedekind ring in an algebraic number field, $i(G, R)$ is known to be the torsion submodule Tor $(a(G ; R))$ of $a(G ; R)$. Thus $x \cdot y=0$ in $a(G ; R)$ for any two torsion elements $x, y \in a(G ; R)$.

\section{REFERENCES}

1. S. B. Conlon, Decompositions induced from the Burnside algebra, J. Algebra 10 (1968), 102-122.

2. A. Dress, On integral representations, Bull. Amer. Math. Soc. 75 (1969), 10311034.

3. - Vertices of integral representations, Math. Z. (to appear).

4. J. A. Green, The modular representation algebra of a finite group, Illinois J. Math. 6 (1962), 607-619.

5. - A transfer theory for modular representations, J. Algebra 1 (1964) 73-84.

6. T. Y. Lam and I. Reiner, Relative Grothendieck groups, J. Algebra (to appear).

7. - Reduction theorems for relative Grothendieck rings, Trans. Amer. Math. Soc. (to appear).

8. - Relative Grothendieck rings, Bull. Amer. Math. Soc. 75 (1969), 496-498.

9. R. G. Swan, The Grothendieck ring of a finite group, Topology 2 (1963), 85-110.

Free University, BerLin, Germany and

Institute for Advanced Study, Princeton, New Jersey 08540 